\title{
Total laparoscopic hysterectomy with previous cesarean section using a standardized technique: experience of Pontificia Universidad Catolica de Chile
}

\author{
C. Celle ${ }^{1} \cdot$ C. Pomés $^{1} \cdot$ G. Durruty ${ }^{1} \cdot$ M. Zamboni ${ }^{1} \cdot$ M. Cuello $^{1}$
}

Received: 14 October 2014 / Accepted: 4 May 2015 /Published online: 15 May 2015

(C) Springer-Verlag Berlin Heidelberg 2015

\begin{abstract}
Total laparoscopic hysterectomy (TLH) in the presence of patients with previous cesarean section (CS) is becoming increasingly common. When performing TLH in these patients, bladder adhesions to the uterus may make dissection much more difficult with higher complication rates. The aim of this study was to assess the safety of TLH in patients with previous CS in an OBGYN residence program. Retrospective study of all TLH performed at our center for either benign or malignant conditions. Of our study cohort, $40 \%$ had undergone one or more previous CS. Average surgical time was $128 \mathrm{~min}$ for patients without previous CS and 136 min for patients with previous CS ( $p=\mathrm{NS})$. Conversion to laparotomy was required in $1 \%$ of cases showing no variation between the CS and non-CS groups. The overall complication rate among patients undergoing TLH was $3.5 \%$. Major complication rate was of $3 \%(n=14), 5$ cases with previous CS and 9 cases with no previous CS ( $p=$ NS). Urologic lesion was the most common major complication, accounting for $1.5 \%(n=7)$ of all cases, 3 cases with previous CS and 4 with no previous CS $(p=\mathrm{NS})$. Of urologic complications, three were cystotomies, 1 with no previous CS and 2 with previous CS ( $p=$ NS). TLH in patients with 1 or more previous CS is technically feasible. In the hands of thoroughly trained laparoscopic surgeons using a standardized technique, it is a safe procedure with minimal complication rates and may be even performed by OBGYN residents ensuring the same success rates.
\end{abstract}

C. Celle

claudiacelle@gmail.com

1 Division of Obstetrics and Gynecology, UC-Christus Health Network, Pontificia Universidad Católica de Chile, Lira 85, 5th floor, Santiago 8330074, Chile
Keywords Total laparoscopic hysterectomy · Cesarean section

\section{Introduction}

Hysterectomy is the most common gynecologic surgery performed worldwide [1]. More than $70 \%$ of hysterectomies are indicated for benign pathologies, mainly uterine fibroids [2]. Three approaches exist to perform hysterectomy: vaginal, abdominal, and laparoscopic. To date, vaginal hysterectomy $(\mathrm{VH})$, when feasible, is the preferred and recommended route for most of cases, especially in an unscarred uterus. A recent Cochrane review stated that $\mathrm{VH}$ should be performed in preference of abdominal hysterectomy ( $\mathrm{AH}$ ) when possible given the significant advantages that the vaginal approach offers [3]. They also concluded that laparoscopic hysterectomy (LH) is associated with less bleeding, similar surgical times, and shorter hospital stays when compared to $\mathrm{AH}$, and therefore, LH should be the preferred route when VH is not possible [3].

Currently, hysterectomy constitutes the second most common operation performed in women after cesarean section (CS) [1], which accounts for up to $60 \%$ of deliveries in some countries as estimated by the recent CORONIS trial [4]. In Chile, the current average rate of cesarean section has reached almost $40 \%$, and it has shown an increasing tendency during recent years $[4,5]$. Moreover, health system statistics show that even as high as a $70 \%$ of pregnancies are delivered through cesarean section in the private clinical setting [5-7]. This rate leaves us as the third country with the highest cesarean rate, among The organization for Economic Co-operation and Development (OECD) members, only preceded by Mexico and Turkey [5]. Thus, the estimated number of patients undergoing hysterectomy with a previous cesarean section is expected to be higher every year. 
All patients undergoing hysterectomy, or any gynecologic surgery, are in risk of suffering complications during and after surgery. In patients with previous CS, these risks are much higher and principally related to major blood loss or urologic injuries [8]. Surgical adhesions caused by previous CS may cause distortion of pelvic anatomy, making the vaginal approach technically difficult and therefore an unsafe procedure. This is of outmost importance when mobilizing the bladder off the cervix, a critical step during any hysterectomy. Thus, making the abdominal route a safer alternative for gynecologic surgeons who have been better trained with open surgery. In fact, surgeons tend to prefer the access route with which they feel more confident and exprienced. Surgical innovation, against a well known and dominated technique, is always considered less safe and guilty of any adverse event observed when introducing the new technique. In addition, no publication has addressed the impact of introducing a standarized laparoscopic technique to evaluate the confidence acquired by surgeons and see how they decide to change their prefered access route for total hysterectomy in patients with previous scar.

With the progressive improvement of instrumentation, the reduction in costs associated to laparoscopy, and the gain of expertise among surgeons, LH has started to replace $\mathrm{AH}$ for several indications [3,9]. A major advantage of laparoscopy is the magnified view and detailed exposure of pelvic anatomy that allows the surgeon to easily recognize and access pelvic structures (for example, vascular pedicles, ureters, and pelvic spaces) reducing the risk of bleeding and inadvertently damaging them during dissection [10]. Thus, the laparoscopic approach should constitute a good alternative for cases with previous CS particularly for surgeons who have become well trained in the technique. Besides offering a better view, the laparoscopic approach also offers advantages in terms of operative time, hospital stay, use of analgesia, and short-term patient satisfaction. Therefore, the safety of this approach for hysterectomy in patients with previous CS should be taken into account and evaluated.

No matter what route is chosen by the surgeon, hysterectomy will be a great challenge in patients with previous pelvic or abdominal surgeries, particularly with previous CS. Technical difficulties will be higher, and so will be the complications. Alternatively, surgeons could prefer to perform a subtotal laparoscopic hysterectomy (STLH) instead of total laparoscopic hysterectomy (TLH) to reduce the risk of urological injuries. So far, there is no evidence to support the election of STLH over TLH in patients with previous CS.

Aware of the importance of an adequate training in laparoscopy before performing any advanced laparoscopic procedure, such as TLH, the obstetrics and gynecology residence program in the school of medicine at Pontificia Universidad Católica de Chile has implemented a progressive and supervised laparoscopic training program. All residents must approve the basic and advanced modules before performing any laparoscopic procedure, especially a TLH. In addition, to reduce intraoperative complications, our division has adopted a standardized technique that is applied to all cases undergoing surgery at our institution.

The aim of this study was to assess the safety of TLH in patients with one or more previous CS compared to those without previous surgery in an institution that has an obstetrics and gynecology (OBGYN) residence program and in which all residents perform a standardized technique under supervision.

\section{Materials and methods}

We conducted a retrospective study of all TLH performed at the Clinical Hospital of Universidad Catolica and San Carlos Clinic, both belonging to the UC-Christus health network. All patients included in the study signed an informed consent, IRB approved, before undergoing TLH based on their medical condition. The data was collected between January 2006 and April 2014.

There were no exclusion criteria based on the number of previous cesarean section deliveries or surgical indication.

As mentioned in the introduction, a standardized surgical technique was used in all cases, with or without previous CS. We performed a lateral approach as described by Sinha et al. since the bladder is not in direct contact with the cervix at this area [11]. Most of the surgery was carried out using only bipolar energy ( $35 \mathrm{~W})$, and we restricted the use of monopolar energy (pure cut, $50 \mathrm{~W}$ ) exclusively to perform the colpotomy in order to reduce thermal damage which has been associated to an increased risk of future vault dehisence [12]. The vaginal vault was then sutured with one single uninterrupted VICRYL-CT Suture (Polyglactin 910) including a uterosacral ligament plicature.

Simultaneously with patient data recollection, we reviewed the delivery database of the health network to register the cesarean rate of each year included in the study to build up the cesarean rate tendency of the period.

Patient demographics, diagnosis, operative time, conversion to laparotomy, uterine weight, and intraoperative and postoperative complications were analyzed. All hysterectomies were performed in a teaching setting, most of them operated by a third year resident assisted by an experienced surgeon who was actively participating of the residence program. All third year residents were required to complete and approve both basic and advanced laparoscopic training modules, during their first and second year residence, respectively, in order to be able to perform a TLH or any other laparoscopic procedure. In order to have an estímate on how many TLH were performed by each resident, they are asked to register every surgical procedure done as first surgeons throughout their complete residency. 
Major complications included ureteric injuries, inadvertent cystostomy, vesicovaginal fistula, bowel injuries, sepsis, vault hematoma, vault abscess, and significant hemorrhage.

Statistical analysis included all patients. Differences between groups with and without previous CS were tested with Chi Square, Fisher's exact test, or Student's $t$ test. A $p$ value $<.05$ was considered statistically significant.

\section{Results}

From January 2006 through April 2014, a total of 458 total laparoscopic hysterectomies were identified. Medical record of prior mode of delivery was available in 454 patients. Forty percent of patients $(n=181)$ had 1 or more previous CS (median 1, range 1-4). The number of previous CS is detailed in Table 1.

A benign condition was the indication for hysterectomy in $85 \%$ of cases and a malignancy for the remaining $15 \%$. Fibroid (symptomatic leiomyoma) was the main indication for hysterectomy accounting for $41 \%$ of cases. Indications for hysterectomy are summarized in Table 2.

The median age of patients was 47 years (median 47, range 30-83). The average surgical time was of $131 \mathrm{~min}$ (median of $120 \mathrm{~min}$, range 50-360 $\mathrm{min}$ ). For the group of patients without previous CS, the average surgical time was $128 \mathrm{~min}$ (median $120 \mathrm{~min}$, range 55-360 min). For patients with previous CS, the average time was 136 min (median 120 min, range 70360) $(p=\mathrm{NS})$. In both groups, we registered cases with longer surgical time. All of them corresponded to complex cases such as uterine or ovarian cancers and extensive or deep infiltrating endometriosis where complete surgical staging or extensive adherence removal was performed, respectively, in addition to TLH. Conversion to laparotomy was required in $1 \%$ of patients $(n=5)$. Three of these patients had 1 or more previous CS ( $p=$ NS, compared to patients without CS). Reasons for conversion were unrecognizable access to the uterine pedicles (1 case with CS), inadequate hemostasis of uterine vessels (1 case with CS), inadequate access to pelvic spaces due to severe adhesions to safely perform nodal staging in a uterine cancer (1 case without CS), severe pelvic adhesions with

Table 1 Previous CS in TLH

\begin{tabular}{lll}
\hline Previous CS & Number of patients & $\%$ \\
\hline 0 & 273 & $60 \%$ \\
1 & 99 & $22 \%$ \\
2 & 61 & $13 \%$ \\
3 & 18 & $4 \%$ \\
4 & 3 & $1 \%$ \\
Total & 454 & $100 \%$ \\
\hline
\end{tabular}

Table 2 Indications for TLH

\begin{tabular}{llll}
\hline Main diagnosis & Without CS \% (n) & With CS \% (n) & $p$ \\
\hline Myoma & $44,3(121)$ & $35(63)$ & 0.054 \\
Adenomiosis & $15(41)$ & $30,4(55)$ & 0.0001 \\
Endometrial cancer & $11,4(31)$ & $3,3(6)$ & 0.004 \\
Endometrial hyperplasia & $5(13)$ & $6,6(12)$ & 0.52 \\
Metrorrhagia & $6(16)$ & $2,2(4)$ & 0.1 \\
Endometriosis & $2,2(6)$ & $5(9)$ & 0.18 \\
Endometrial polyp & $2,6(7)$ & $3,4(9)$ & 0.27 \\
Prolapse & $1(3)$ & $1,1(2)$ & 1.0 \\
Others & $12(33)$ & $11,6(21)$ & 0.99 \\
Total & $100(273)$ & $100(181)$ & \\
\hline
\end{tabular}

extensive attachment of the uterus to rectosigmoid colon (1 case without CS), and repair of a urological lesion (1 case in a patient with 3 previous CS).

The overall complication rate among patients undergoing TLH was $3.5 \%$. Major complication rate was of $3 \%(n=14)$, 5 cases with previous $\mathrm{CS}$ and 9 cases with no previous $\mathrm{CS}(p=$ NS). Urologic lesion was the most common major complication, accounting for $1.5 \%(n=7)$ of all cases (Table 3$), 3$ cases with previous CS and 4 with no previous CS $(p=\mathrm{NS})$. Regarding urologic complications, three were cystotomies, 1 with no previous CS and 2 with previous CS ( $p=$ NS).

There were 9 intraoperative complications. Three patients with cystotomy diagnosed and repaired during surgery (two laparoscopically and one after conversion to laparotomy). One of these cases developed a vesicovaginal fistula that was diagnosed shortly after removing the Foley catheter. She was asymptomatic and was managed conservatively showing complete sealing after 2 months of follow-up. Other two patients experienced ureteral thermic lesions adverted during surgery that required double $\mathrm{J}$ stent installation, postoperatively. Two patients with uncontrollable bleeding required conversion to achieve adequate hemostasis. Another patient presenting extensive subcutaneous emphysema required to stop the surgery and complete it via vaginal access. Finally, a patient with a rectal lesion repaired uneventfully during the same
Table 3 Major complications of TLH

\begin{tabular}{ll}
\hline Type of complicaction & Number \\
\hline Ureteric injury & 4 \\
Inadvertent cystotomy & 3 \\
Bowel injury & 1 \\
Vesicovaginal fistula & 2 \\
Vault hematoma & 1 \\
Vault abscess & 1 \\
Significant hemorrhage & 3 \\
Total & 14 \\
\hline
\end{tabular}


intervention. No significant difference in terms of intraoperative complication rate was observed between the two groups (see Table 4).

There were 8 postoperative complications. Two were diagnosed immediately after surgery, at the recovery room. The first case, a patient with massive intra-abdominal bleeding and hypovolemic shock reoperated and recovered without further adverse events. The second case, a patient with an atrial fibrillation handled successfully with external cardioversion with no further intervention. Late postoperative complications are described later on. No significant difference in terms of postoperative complication rate was observed between the two groups (see Table 4).

The average postoperative hospital stay was of 3 days (range 2-6 days) interchangeably between patients with or without previous CS. No difference in hospital stay was observed between groups.

Pathologic analysis demonstrated similar distribution of diagnosis between groups. The average uterine weight was $155 \mathrm{~g}$ for both groups (range 30-1170 g). No difference was observed in uterine weight between groups.

There were $9(2 \%)$ readmissions. Four of them were due to postoperative fever. In three cases, a vault abscess was diagnosed, and all were discharged in good conditions after completion of their antibiotic treatment. The remaining patient presented with fever of unknown origin, with normal blood tests and normal abdomen/pelvis CT scan, who was discharged in good conditions after completing her antibiotic treatment. Two cases corresponded to inadvertent ureteral lesions that presented with acute abdomen due to uroperitoneum (approximately 2 weeks after surgery). Both cases were successfully managed with ureteral reimplantation. Another readmission corresponded to a patient with vaginal discharge, starting 1 week after surgery, who was diagnosed with a vesicovaginal fistula. She was initially managed with permanent Foley catheter, and after 2 months of use, she underwent successful surgical repair of her fistula. Worth mentioning, we had 1 more case of vesicovaginal fistula that originated from a cystotomy repaired during TLH surgery. This case was successfully managed, in an ambulatory setting, with prolonged bladder catheterization until complete fistula sealing with no further need of surgery. Finally, the last two readmission cases corresponded to a case of acute pyelonephritis (treated with

Table 4 Surgery characteristics and postoperative stay

\begin{tabular}{llll}
\hline Characteristics & Without CS & With CS & $p$ \\
\hline Surgical time (min) & 128 & 136 & 1.0 \\
Intraoperative complications $(n)$ & 4 & 5 & 0.494 \\
Postoperative complications $(n)$ & 7 & 1 & 0.15 \\
Postoperative hopital stay (days) & 2,5 & 2,5 & 0.61 \\
\hline
\end{tabular}

antibiotic regimen) and a case of vault hematoma (managed with oral analgesia).

In this study, we also included patient data from the San Carlos Clinic where normally, residents do not carry out clinical rotations. This center covers 12 to $15 \%$ of all our TLH procedures. Overall, once accounting for the total number of TLH perfomed by each resident, we estimated that about 85 $90 \%$ of our TLH were performed by a third year resident as first surgeon, and the remaining 10-20\% performed exclusively by 2 experienced laparoscopic surgeons.

\section{Discussion}

In the present paper, we demonstrated that laparoscopic approach for total hysterectomy in patients with previous cesarean section is a safe procedure and that our results are comparable to other series already published (see Table 5) [11, 13]. The use of a standardized technique accelerates the learning curve and minimizes surgical risks, thus allowing us to offer the best surgical intervention possible indistinctively to all patients, regardless of the previous number of CS. This is important, especially in a country like Chile where the rate of CS is one of the highest reported to date worldwide and where local health authorities have not been yet able to find a solution in order to revert this worrisome tendency.

It is a fact that TLH in the presence of patients with previous $\mathrm{CS}$ is becoming increasingly common since the number of cesarean deliveries is increasing worldwide [5]. This might pose a higher difficulty for surgeons approaching TLH since bladder adhesions to the uterus may make dissection much more difficult and might even preclude bladder mobilization off the cervix. In our series, all TLH were successfully performed with the lateral approach to the space. Of these, only $13 \%(n=54)$ were vaginally assisted due to surgeon expertise. When adjusting by year, more than $60 \%$ of these cases were performed before year 2010 supporting the learning curve of the laparoscopic technique. The inclusion of standardized techniques in the learning process of TLH, or any surgery for that matter, accelerates the learning curve. This only puts to evidence that our surgeons have acquired the necessary

Table 5 Complication comparisson between publications

\begin{tabular}{llll}
\hline Publications & $\begin{array}{l}\text { TLH with } \\
\text { CS }(\%)\end{array}$ & $\begin{array}{l}\text { Inadvertent } \\
\text { cystotomy } \\
\text { with CS }(\%)\end{array}$ & $\begin{array}{l}\text { Total major } \\
\text { complication } \\
\text { with CS }(\%)\end{array}$ \\
\hline Wang et al. & $141(24,4)$ & $7(5)$ & $20(14,2)$ \\
Sinha et al. & $261(100)$ & $2(0,8)$ & $\mathrm{n} / \mathrm{a}$ \\
Celle et al. & $181(40)$ & $2(1,1)$ & $5(2,8)$ \\
Total & 583 & $11(2)$ & $\mathrm{n} / \mathrm{a}$ \\
\hline
\end{tabular}

n/a data not available 
Table 6 Major complications of TLH according to number of previous CS

\begin{tabular}{llllll}
\hline Type of complicaction & $1 \mathrm{CS}(N=99)$ & $2 \mathrm{CS}(N=61)$ & $\geq 3 \mathrm{CS}(N=21)$ & NO CS $(N=273)$ & Total \\
\hline Ureteric injury & 1 & 0 & 0 & 3 & 4 \\
Inadvertent cystotomy & 1 & 0 & 1 & 1 & 3 \\
Bowel injury & 0 & 0 & 0 & 1 & 1 \\
Vesicovaginal fistula & 1 & 0 & 0 & 1 & 2 \\
Vault hematoma & 0 & 0 & 0 & 1 & 1 \\
Vault abscess & 0 & 1 & 0 & 2 & 1 \\
Significant hemorrhage & 1 & 0 & 0 & & 14 \\
Total & & & & 0 & 3 \\
\hline
\end{tabular}

skills in order to feel confident completing the totality of this procedure through the laparoscopic access, having no further need of assistance through the vaginal approach thus reducing surgical times, risks, and costs of adding further surgical equipment (vaginal and laparoscopic). In our center, the use of this standardized technique has determined that the number of previous CS has no detrimental influence in the surgical outcome (see Table 6). In fact, no difference was observed in the number of major complications when comparing TLH carried out in patients with 1 versus $\geq 3$ previous CS. Accordingly, before year 2010, around 20-30\% of our TLH had 1 or more previous CS, whereas to date, 40 to $50 \%$ of our patients have one or more previous CS, and yet, our TLH procedures have not diminished. Figure 1 shows the percentage of previous CS for every TLH performed each year. In addition, for the same period of time, a sustained reduction in the number of abdominal hysterectomies and subtotal hysterectomies has taken place. More importantly, surgeons that used to prefer a subtotal or total abdominal hysterectomy for this type of patients currently choose TLH as first option after learning the standardized technique. Nowadays, there is no longer contraindication for TLH based on number of previous CS in our institution; we just ask to have a well-trained team.

Urinary tract injuries are the most feared complications in gynecologic surgery. They account for $1 \%$ of all gynecologic procedures [8]. The series by Donnez et al. shows a $0.56 \%$ of urinary tract injuries and a $1.59 \%$ of overall complications [14]. Our overall complication rate was of $3.5 \%$ with a $1.5 \%$ of urologic lesions. As we mentioned earlier, no statistical difference was observed between groups. A major concern for surgeons, when performing TLH in patients with one or more previous CS, would be the risk of bladder injury. Rooney et al. demonstrated that previous cesarean section is
Fig. 1 Evolution in the number of TLH with or without previous CS and the percentage of cesarian section per year at the Pontificia Universidad Catolica de Chile between 2006 and 2014

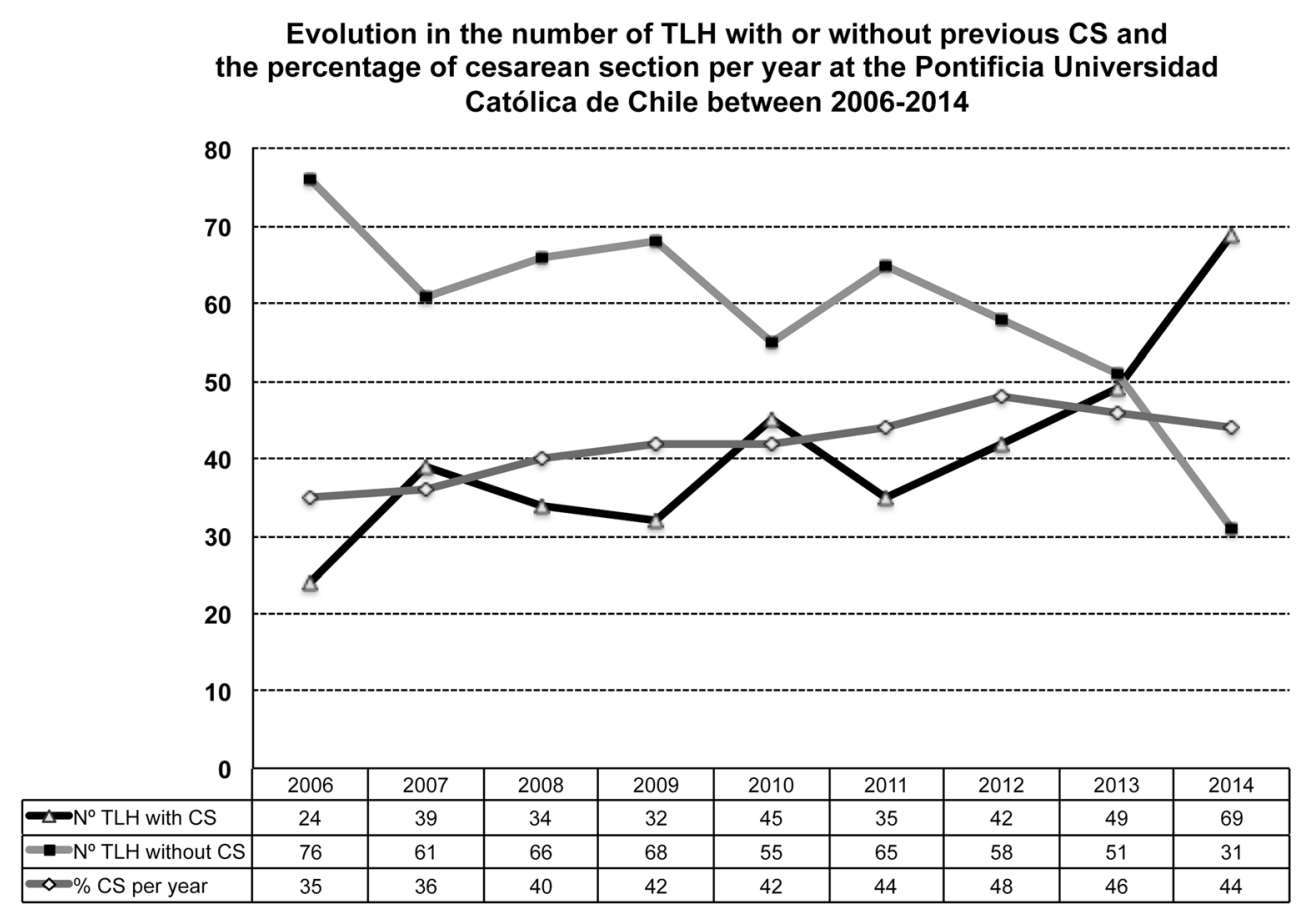


a significant independent risk factor for lower urinary tract damage, reporting a $1 \%$ incidental cystotomy rate [15]. Pillet et al. also reported a $1 \%$ rate of bladder injury in their TLH series and found that previous CS as well as previous laparotomy were the main risk factors associated to bladder injury [16]. We had a $0.7 \%(n=3)$ of cystotomies, all of them repaired in the same surgical act.

Assuming then that the risk of cystotomy should be higher in patients with increasing number of previous $\mathrm{CS}$, we further analyzed major complication rate occurrence in the subgroup of patients with 1,2, and 3 or more previous CS. We observed that the number of previous scars had no relation to the number or type of complication (Table 6). Under this same assumption, surgical operative time should be longer due to the major surgical challenge associated to numerous scars. Average surgical time was of $140 \mathrm{~min}$ in patients with 1 previous CS, 130 min with 2, and 136 min for patients with 3 or more previous CS showing that this was not a conditioning factor regarding operative time.

Based in our results and others, we believe that there is no justification for subtotal hysterectomy, in most of cases, as an alternative to TLH in order to reduce urological complications. Technically speaking, for many surgeons, subtotal laparoscopic hysterectomy (STLH) could be easier to perform since no bladder dissection and descent are required to remove the uterine corpus. Thus, a reduced complication rate should be expected with this approach. However, the evidence has showed that retaining the cervix does not offer advantages in regards to complication rate, bladder symptoms or incontinence, improvement of libido, and bowel dysfunction [1, 17]. More importantly, we must recall that by preferring TLH, where the cervix is removed, there is no risk of future bleeding as seen occasionally in subtotal hysterectomy cases [17].

We believe that a major determinant of our complication rate is based on the training program and the standardized technique that we have incorporated at our institution. All our residents, fellows, and teaching surgeons have completed the same laparoscopic training program and perform the same hysterectomy technique. Completion of the training program and learning curve, adequate case selections for our trainees to start operating, and the appropriate supervision through a gradual increase in difficulty guarantee the success rate. In fact, following this educational strategy, about $90 \%$ of TLH are currently operated by a third year fellow with the assistance of a teaching surgeon and with low complication rate.

However, some limitations exist to the analysis reported in our study. First, although we tried to control for all patient and surgical variables that could potentially increase surgical difficulty, this was a retrospective study, and as such, we were unable to recollect information on patient BMI and uterine size, two known factors that could independently increase the difficulty of hysterectomy. Second, our data included cases where the learning curve was taking place. The fact that almost all our procedures are performed at a teaching institution by a third year resident might have influenced on surgical timing and complications despite the fact that they were not statistically significant between groups. In a private clinical setting, bladder adhesions secondary to previous CS might pose much less of a challenge to an experienced surgeon comfortable using sharp dissection regularly.

To date, the current published literature on TLH in patients with previous CS is scarce and limited. To our knowledge, this is the first study that evaluates the feasibility of performing TLH in patients with previous CS performed predominantly by fellows in their OBGYN residence program. We firmly believe that TLH offers multiple advantages over any other approach. Our findings support the safety of TLH in patients with previous CS. The use of a standardized technique allows trainees to perform this surgery safely with minimal complication rates.

Conflict of interest These data have not been published or presented elsewhere, and the manuscript is not under review at any other journal. This study has been currently presented orally in the ESGE 2014 congress held in Brussels. On behalf of all authors, the corresponding author states that there is no conflict of interest.

Informed consent Informed consent was obtained from all patients for being included in the study.

\section{References}

1. Sutton C (2010) Past, present, and future of hysterectomy. J Minim Invasive Gynecol 17:421-435. doi:10.1016/j.jmig.2010.03.005

2. Whiteman MK, Hillis SD, Jamieson DJ et al (2008) Inpatient hysterectomy surveillance in the United States, 2000-2004. Am J Obstet Gynecol 198:34.e1-7

3. Nieboer TE, Johnson N, Lethaby A, Tavender E, Curr E, Garry R et al (2009) Surgical approach to hysterectomy for benign gynaecological disease. Cochrane Database Syst Rev. doi: 10.1002/14651858.CD003677.pub1

4. The CORONIS Collaborative Group (2013) Caesarean Section surgical techniques (CORONIS): a fractional, factorial, unmasked, randomised controlled trial. Lancet 382:234-248. doi: 10.1016/S0140-6736(13)60441-9

5. OECD. Health Statistics 2013. OECD Publishing, 10.1787/healthdata-en

6. Departamento de Estadística e Informática del Ministerio de Salud, Instituto Nacional de Estadísticas, Atenciones del sector privado, REMSAS 2011. http://intradeis.minsal.cl/reportesremsas/2011/ partos abortos/partos abortos.aspx. Published 2011. Accessed 19 Aug 2014

7. Guzmán E (2012) Epidemiological profile of caesarean section in Chile in the decade 2000-2010. Medwave 12(3), e5331. doi:10.5867/medwave.2012.03.5331

8. Bai SW, Huh EH, Jung DJ, Park JH, Kim SK, Park KH (2006) Urinary tract injuries during pelvic surgery: incidence rates and predisposing factors. Int Urogynecol J 17:360-364 
9. Jacoby VL, Autry A, Jacobson G, Domush R, Nakagawa S, Jacoby A (2009) Nationwide use of laparoscopic hysterectomy compared with abdominal and vaginal approaches. Obstet Gynecol 114: 1041-1048. doi:10.1097/AOG.0b013e3181b9d222

10. Walsh CA, Walsh SR, Tang TY, Slack M (2009) Total abdominal hysterectomy versus total laparoscopic hysterectomy for benign disease: a meta-analysis. Eur J Obstet Gynecol Reprod Biol 144: 3-7. doi:10.1016/j.ejogrb.2009.01.003

11. Sinha R, Sundaram M, Lakhotia S, Hedge A, Kadam P (2010) Total laparoscopic hysterectomy in women with previous caesarean sections. J Minim Invasive Gynecol 17:513-517. doi:10.1016/j.jmig. 2010.03.018

12. Cronin B, Sung V, Matteson K (2012) Vaginal cuff dehiscence: risk factors and management. Am J Obstet Gynecol 206(4):284-288. doi:10.1016/j.ajog.2011.08.026

13. Wang L, Merkur H, Hardas G, Soo S, Lujic S (2010) Laparoscopic hysterectomy in the presence of previous caesarean section: a review of one hundred forty-one cases in the Sydney West Advanced Pelvic Surgery Unit. J Minim Invasive Gynecol 17:186-191. doi: 10.1016/j.jmig.2009.11.007
14. Donnez O, Jadoul P, Squifflet J, Donnez J (2009) A series of 3190 laparoscopic hysterectomies for benign disease from 1990 to 2006: evaluation of complications compared with vaginal and abdominal procedures. BJOG 16(4):492-500. doi:10.1111/j.1471-0528.2008. 01966.x

15. Rooney CM, Crawford AT, Vassallo BJ, Kleeman SD, Karram MM (2005) Is Previous cesarean section a risk for incidental cystotomy at the time of hysterectomy?: A case-controlled study. J Minim Invasive Gynecol 193:2041-2044. doi:10.1016/j.ajog.2005.07.090

16. Lafay Pillet MC, Leonard F, Chopin N, Malaret JM, Borghese B, Foulot H, Fotso A, Chapron C (2009) Incidence and risk factors of bladder injuries during laparoscopic hysterectomy indicated for benign uterine pathologies: a 14.5 years experience in a continuous series of 1501 procedures. Hum Reprod 24:842-849. doi:10.1093/ humrep/den 467

17. Lethaby A, Mukhopadhyay A, Naik R (2012) Total versus subtotal hysterectomy for benign gynaecological conditions. Cochrane Database Syst Rev. doi:10.1002/14651858.CD004993.pub3 